The Role of A/B Tests in the Study of Large-Scale Online Learning

\author{
Alexander O. Savi \\ University of Amsterdam \\ Joseph J. Williams \\ Harvard University \\ Gunter K. J. Maris \\ Cito Institute for Educational Measurement, University of Amsterdam \\ Han L. J. van der Maas \\ University of Amsterdam
}

\begin{abstract}
Author Note
Correspondence concerning this preprint should be addressed to Alexander Savi, University of Amsterdam, Department of Psychological Methods, Postbus 15906, 1001 NK, Amsterdam, The Netherlands. Email: o.a.savi@gmail.com. Funding by Netherlands Organisation for Scientific Research, grant number CI1-12-S037.
\end{abstract}

\begin{abstract}
Although large-scale online learning increasingly succeeds in attracting learners worldwide, to date it fails to deliver on its promise. We first show the immense popularity of online learning and discuss its (unsatisfactory) effectiveness. We then discuss large-scale online randomized controlled experiments ( $\mathrm{A} / \mathrm{B}$ tests) as a powerful complimentary means to enable the desired leap forward. Although these experiments are widely and intensively used for web page optimization, and are slowly being adopted by the online learning community, their use, benefits,
\end{abstract}


and challenges have only limitedly seeped through to the larger learning community. We summarize existing efforts in employing A/B tests in online learning, argue that such tests should take into account the typical nature of (online) learning, and encourage the use of knowledge from the various learning sciences to identify interventions that promise improved learning. We finally discuss both the limitations and promises of A/B tests, and show how such tests can ultimately contribute to learning that is tailored to each individual learner. The insights and priorities that arise from this overview and synthesis of $\mathrm{A} / \mathrm{B}$ tests in online learning may help advance and direct the field.

Keywords: online learning, online experimentation, randomized controlled trials, learning interventions, research methodology 


\section{The Role of A/B Tests in the Study of Large-Scale Online Learning}

Online learning is sometimes attributed, rather boldly, the power to solve the 2 sigma problem: the finding that learners that are tutored one-to-one perform two full standard deviations better than learners that receive conventional instruction (Bloom, 1984). Although this is not its only promise, and 2 sigma likely is an overestimation (VanLehn, 2011), it does sets a challenge. Indeed, online learning's large scale, easy adaptability, and inference from its generously generating data, are argued to enable unprecedented optimization and personalization and significantly increase learning gains. To date, however, the majority of online learning seems to fail to convincingly deliver on its promise, and both researchers and (online) educators seek to discover which components actually do make it truly effective. In this paper, we motivate the urgent need to prioritize the effectiveness of online learning and discuss a largescale online experimental approach that is slowly being adopted by some providers of large-scale online learning, and that may potentially provide a leap forward. We ultimately argue that this approach is not just a powerful way to greatly increase the effectiveness of online learning, but also an opportunity to expand our knowledge of effective components in learning and education as a whole.

In the first section, we define large-scale online learning (hereinafter simply referred to as online learning) and show its rapidly increasing popularity, followed by a discussion of its disputed effectiveness. This should not only introduce less familiar readers to the field of online learning, but also convey the urgency to prioritize its return on investment. Return on investment may be defined as the increased achievement with respect to devoted time, or as the increased aspiration or perseverance in learning. We are however reluctant to pinpoint its definition, as it is greatly determined by one's personal educational philosophy and associated 
goals.

In the second section, we discuss the use of $\mathrm{A} / \mathrm{B}$ tests (large-scale online randomized controlled experiments) to identify and iteratively optimize learning interventions for the online environment. We give examples of existing efforts to employ such experiments, followed by an extensive discussion of its most pertinent requirements and accompanying challenges. Finally, we discuss its limitations. Again, this should not only provide less familiar readers with a brief overview of the field of A/B testing in large-scale online learning, but should also locate pressing issues that need to be dealt with in order to advance the field.

\section{A Primer on Online Learning}

Online learning comes in a great variety. It ranges from learning activities that resemble traditional education such as massive open online courses (i.e., xMOOCs, such as Coursera, edX, FutureLearn, and Udacity), to a plethora of activities characterized by a practice-approach and the use of various gamification elements (e.g., Codecademy, Duolingo, Khan Academy, KnowRe, and Math Garden), and ultimately full-fledged games that promote implicit learning through interaction with the game mechanics (e.g., DragonBox Algebra, Geniverse, Mars Generation One: Argubot Academy, Mathbreakers, Wuzzit Trouble, and Zoombinis). These activities may take place entirely online or blended with traditional education (e.g., Molnar, 2013), range from learning limited concepts (e.g., Slice Fractions, and Vax!) to earning complete degrees (e.g., Minerva Project, and Udacity's Online Master of Science in Computer Science in collaboration with Georgia Tech and AT\&T), cover many subjects relevant from kindergarten to higher education, and are too used across virtually all ages.

We reflect this variety by defining large-scale online learning as any learning activity that is provided online and that scales. Large-scale online learning may thus be seen as an umbrella 
term for similar and related concepts such as blended/hybrid learning, distance learning, elearning, intelligent tutoring systems, massive open online courses (cMOOCs and xMOOCs), and serious games, provided that these obey the terms in our definition. Naturally, lumping these together seems to do no justice to the rich history and diversity of each of the individual concepts, and in many contexts prudence is called for when comparisons between them are being made. However, in the context of the current paper it nicely illustrates the broad applicability of the experimental approach that is proposed and discussed in the second section.

\section{The Rise and Rise of Online Learning}

The number of users of online learning has rapidly increased the past few years. Although accurate figures are sparse, many of those figures pertain solely to the U.S., and moreover do not equally represent the whole spectrum of online learning, the few figures that are available do give a compelling picture of the popularity of online learning (a global group of organizations called 'GlobalOHER initiative' aims to provide survey-based figures on online higher education worldwide (ICDE, 2013), however no report was released yet). In the following we summarize these figures and purposely echo the terms used for online learning in their respective sources, since each may pertain to some class of online learning and not necessarily to the whole of online learning.

To begin with primary and secondary education (roughly aged 4 to 18 ), a survey by the private Evergreen Education Group (Watson, Murin, Vashaw, Butch, \& Rapp, 2013) estimates that 310.000 U.S. students were enrolled in fully online schools in $2012 / 2013$. Another survey by the U.S. National Center for Education Statistics (NCES) shows that enrollment of U.S. high school students (roughly aged 16 to 18 ) in distance education courses has rapidly increased over the past years (Aud et al., 2012). In 2002/2003 roughly 222.000 students enrolled, which 
increased to 310.000 students in 2004/2005, and 1.3 million students in 2009/2010. Twelve percent of the school districts serving these students in 2009 to 2010 reported that these students could fulfill all requirements for graduation with distant courses. Finally, a survey on teaching with digital games by the Joan Ganz Cooney Center estimates 74\% of K-8 teachers to use digital games for instruction (Takeuchi \& Vaala, 2014).

In fall 2012, the NCES also started collecting enrollment figures of U.S. post-secondary students (roughly aged 18 and above) in distance education courses (NCES, 2012) and has issued its first report in 2014 (Ginder and Stearns, 2014, and summarized in Kena et al., 2014). The figures show that, in fall 2012, 2.6 million students were enrolled exclusively in these courses, whereas another 2.8 million students were enrolled in some but not all of these courses. The private Online Learning Consortium (former Sloan Consortium) also provides yearly estimates of the students of online learning in higher education in the U.S. In their 2013 report they estimate a total number of 7.1 million students (Allen \& Seaman, 2014), although some argue that this might be an overestimation (Kolowich, 2014).

Also, the self-reported worldwide coverage of some large providers of online learning adds to this picture of popularity. For instance, the xMOOC provider Coursera reports to serve 7.5 million students worldwide and offer courses from 100 institutions ( $\mathrm{Ng}, 2014)$, including leading universities such as Stanford and Yale. Codecademy, a platform for learning to code, reports to serve over 24 million students worldwide (Sims, 2014). Finally, Khan Academy, a video tutorial and practice platform for a multitude of subjects, reaches over 10 million unique students per month (Murphy, Gallagher, Krumm, Mislevy, \& Hafter, 2014).

Naturally, learning is not limited to the academy. Consultants from Roland Berger, a large strategy consultancy firm, estimate that in Europe 3000 companies are involved in e- 
learning (Vernau \& Hauptmann, 2014), and they expect an average increase of 13\% per year. Also, Udacity and its spin-off Nanodegree partner with companies to build and provide courses specifically tailored to future employees (Chafkin, 2013). Other examples are edX Professional Education, HBX of Harvard Business School, the French First Business MOOC, and the IOC Athlete MOOC by the International Olympic Committee. Finally, also governments have started adopting online learning, with the OpenupEd initiative in the European Union and Coursera's several governmental partnerships (Coursera, 2014a, 2014b) being notable examples.

\section{All That Glitters Is Not Gold}

Unfortunately, all that glitters is not gold. Here we briefly give a few examples from right across the spectrum of online learning, showing some of the struggles different online educators face in optimizing the return on investment of learning. As a first illustration: Sebastian Thrun, founder of Udacity, already warned in 2012 that his MOOCs were experimental and that he had not "seen a single study showing that online learning is as good as other learning" (Lewin, 2012). He substantiated this claim in 2013, when he revealed that despite Udacity's efforts to teach and engage students with courses that use quizzes and gamification techniques, only 7\% managed to finish them (Chafkin, 2013).

Another example comes from Khan Academy: Greenberg, Medlock, and Stephens (2011) found that Khan Academy's lecture videos, a core component of the platform, were mostly avoided by its users. Instead of watching the videos, many users sought help from peers and teachers, and used hints from the platform. Both Coursera and Udacity, xMOOC providers that likewise heavily rely on videos, later confirmed this finding on their respective platforms (Simonite, 2013).

On the other end of the spectrum of online learning struggles are apparent as well. 
Although incomparable to MOOCs and on a decidedly different scale, in a synthesis of some of the research of the Community College Research Center the issue of low retention is also noticed in online courses in community colleges (Jaggars, Edgecombe, \& Stacey, 2013). Moreover, they show that performance outcomes were lower for online than traditional courses, that students who took online courses were less likely to return the following semester, and that the more online courses they took the less likely they were to obtain a degree or transfer to a four-year institution. Additionally, achievement gaps that existed in traditional courses increased in online courses.

A slightly more recent study by the Public Policy Institute of California (Johnson \& Mejia, 2014), also in community colleges, challenged the finding that students taking online courses are less likely to obtain a degree. Although in the short term similar disappointing results were found as by CCRC, results show that in the long-term students that take an online course are more likely to obtain a degree or transfer to a four-year institution than students that only take traditional courses. A multitude of explanations may account for increased long-term outcomes. Students may for instance enrich their curriculum with online courses such that they are better prepared for further education, or it might simply be the most motivated students that take online courses. As the authors put it: "[f]or some students, online courses offer a useful tool that helps them reach their goals". Naturally, this does not necessarily mean that the online courses are effective on their own and the short-term results may signal that online courses, with their lower completion rates and larger achievement gaps, under-perform to traditional courses.

Possibly disappointing findings such as the few we summarize here naturally influence the perceived promise of online learning. This is for instance reflected in the aforementioned survey by the Online Learning Consortium (Allen \& Seaman, 2014), which showed that, 
compared to 2012, in 2013 proportionally less academic leaders indicate that "online learning is critical to their long-term strategy" and proportionally more academic leaders think that "the learning outcomes for online education are inferior to those of face-to-face instruction".

Moreover, only a small minority believes that "there will no longer be concerns about the relative quality of online courses".

Summarizing, we have seen that learners and educators worldwide rapidly adopt online learning, and that companies and countries jump on the bandwagon. To date online learning has enabled affordable learning activities for a broad public and many around the world already benefit greatly. We have however also seen that its effectiveness leaves a lot to be desired. Taking together its inevitable growth, its according impact and role within education as a whole, and an unarguable need to increase its effectiveness, necessitates the use of a robust and reliable method to identify interventions that promise improved learning.

\section{An Experimental Approach to Improve Online Learning}

In this section, we focus on one such method. We discuss the use of large-scale online randomized controlled experiments (i.e., A/B tests): a within-platform approach for iterative and incremental improvement of large-scale online learning. The popularity (and accordingly large scale) of particular online learning environments calls, at least partly, for such an online-only and within-platform approach. Moreover, we focus on A/B testing as we think it is an opportunity that receives too little attention, in spite of its broad applicability. The use of such experiments within the online learning community is quite limited, especially in comparison to web page optimization outside of the online learning context, and also not well-known in the larger educational community. And although it will not turn all glitters into gold, it certainly is a powerful complimentary method that may potentially provide a significant contribution. In the 
following we introduce the use of $\mathrm{A} / \mathrm{B}$ tests, give examples of existing efforts to employ such experiments in online learning, discuss important challenges that are specific to the study of online learning, and meet the inevitable limitations of the method.

\section{A/B Testing}

Both educational practice and research benefit greatly from randomized experiments (Slavin, 2002). Traditionally, different methods are used for varying levels of (causal) inference, such as small-scale laboratory experiments on precise learning interventions, and large-scale randomized controlled trials (RCTs) for comparing distinct educational methods. In online learning, the $\mathrm{A} / \mathrm{B}$ test (also known as 0/1 test, bucket test, content experiment, parallel flight, and split test) provides us with an online RCT that is intensively used in website optimization and that is both convenient and robust. In a typical A/B test different variations of the same web page are shown to different randomly chosen groups of visitors. After a specified amount of time, the groups are compared with respect to an evaluation criterion (Kohavi, Longbotham, Sommerfield, \& Henne, 2009). In other words, in an A/B test a large number of visitors is randomly assigned to a control or treatment variant in order to discern the effects of the treatment variant. Such an experimental test is particularly strong since it enables the establishment of causal rather than correlational relationships. Moreover, $\mathrm{A} / \mathrm{B}$ tests can be extended to involve more than two conditions (i.e., $\mathrm{A} / \mathrm{B} / \mathrm{n}$ tests) or additional variables (i.e., multivariate tests).

Conducting A/B tests reliably requires a tremendous number of users (Kohavi, Deng, Longbotham, \& Xu, 2014), however in the first section we saw that online learning can actually provide that scale. The opportunity this opens up for learning research is unprecedented. In traditional learning mass experimentation (in the form of traditional RCTs) is troublesome and costly, whereas the advent of online learning offers plenty of opportunity for cheap mass 
experimentation. Moreover, in an online environment it is not only much easier to allocate learners randomly, but also to readily and homogeneously implement the experimental interventions.

Finally, it is important to stress that A/B tests differ with respect to one more essential aspect from their offline randomized controlled counterpart. Whereas traditional RCTs for instance enable comparisons between situations that may differ quite significantly (with all accompanying challenges, e.g., Norman, 2003), A/B tests on the other hand are best suited for the comparison of more precise interventions, as its online nature enables very homogeneous and precise adjustments. A/B tests are characterized as minimally invasive (Heffernan \& Heffernan, 2014), and enabling iterative improvement (Williams et al., 2014). Summarizing, on the one hand $\mathrm{A} / \mathrm{B}$ tests share their precision and ease of randomized allocation and double-blinding with traditional laboratory experiments, whereas they share the scale and ecological validity with traditional randomized controlled trials.

\section{Existing Efforts}

Although the (reported) use is very limited, some online educators are already performing A/B tests, or are currently preparing their frameworks to enable the use of these tests (e.g., Coursera (Novet, 2013), Duolingo (Ungerleider, 2014), edX (“Content Experiments,” n.d.), Khan Academy (Rowan, 2013), Udacity (Simonite, 2013)). At Coursera they discovered, using large-scale A/B tests, that students' engagement dropped when those students were reminded of their homework, whereas a reminder of their previous activities increased engagement, and at Udacity they discovered that black and white rather than colorized lessons gave better test results (more details were not provided; Simonite, 2013). At Khan Academy, they discovered that showing a sneak peek of more advanced content demotivated learners to continue studying (Fox, 
2014), that a mindset intervention in the header of math problems increased mastery of those math concepts (Williams, Paunesku, Haley, \& Sohl-Dickstein, 2013), and they have tested different models for assessing students' proficiencies (Hu, 2011). At Duolingo they discovered that introducing the English pronoun 'it' too early in the curriculum confused Spanish students as the word is sometimes used differently in both languages (Stevenson, 2014). Finally, quite some A/B tests were conducted on both the edX framework (some of which were summarized by Reich, 2015) and the ASSISTments tutoring system (Heffernan \& Heffernan, 2014).

Unfortunately, results from A/B tests such as summarized here are rarely found, especially in the scientific literature. Given the recent efforts among online educators to incorporate A/B tests, we do however predict an increase in, hopefully publicly available, studies.

\section{Tailoring A/B Tests to Online Learning}

$\mathrm{A} / \mathrm{B}$ tests have great potential for determining and increasing the effectiveness of online learning, however blindly adopting A/B tests from the domain of website optimization without taking into account the specificities of online learning will fail in fully exploiting that potential. A/B tests are often used in website optimization for relatively simple tweaks, such as textual or graphical changes to for instance the landing page of a retail website. The overall evaluation criterion (i.e., dependent or outcome variable) is in its turn usually expressed as a conversion rate, which is the rate to which visitors or users act in some desired way; this may be the rate to which visitors for instance buy a product from the retail website, or in case of online learning the rate to which users study the course material, watch an instructional video, or continue to practice math problems. However, although those relatively simple interventions may indeed increase the effectiveness of a learning experience, presumably the real progression comes from interventions that are both not that easily implemented and varied, and not that easily captured in 
a conversion metric (such as measures of transfer (Barnett \& Ceci, 2002)). Rather, maximizing the return on investment of online learning has two important requirements. First it requires determining and tracking each learner's position on an educational map, and second it requires offering individualized navigation towards an educational goal (Savi, van der Maas, \& Maris, 2015).

For the first requirement, determining a learner's position, one accessible and accordingly popular method is to use proxy measures for learning, such as frequency of play, time on task, or proportion correct. However, such proxy measures carry important challenges, with the most pressing one being which measures best capture learning (Reich, 2015). On top of that, we deal with a variable that is latent (e.g., ability, learning) rather than manifest (e.g., profit, conversion rate), and one that is inherently subject to change, requiring a microgenetic method (Siegler \& Crowley, 1991).

Powerful yet involved methods that can fulfill the above requirements include Intelligent Tutoring Systems (ITS) and Item Response Theory (IRT). One excellent example of the successful implementation of the latter, in a large-scale educational context, is Math Garden's computer adaptive math practice platform (Klinkenberg, Straatemeier, \& Van der Maas, 2011). Math Garden uses an IRT approach to continuously estimate and update the abilities of learners and difficulties of problems, and an Elo Rating System (ERS) to adaptively match learners with problems that have the desired difficulty.

The proper implementation of such methods is far from trivial and may not be applicable in each and every online educational method. However, tracking or monitoring a learner's development through these and similarly sophisticated methods can open up new opportunities for measurement (e.g., Brinkhuis, Savi, Coomans, Hofman, van der Maas, \& Maris, 2015), as 
these enable measuring the development of each individual learner and estimating the idiosyncratic effects of an intervention.

For the second requirement, individualized navigation towards an educational goal, accurate tracking is again very beneficial. Tracking each learner's development, and identifying for instance their misconceptions or level of expertise, can aid in adapting interventions to individuals, as we have to deal with both individual and contextual differences. For instance, cognitive variability is notoriously large (Siegler, 1994) and may contribute to considerable individual differences. Such differences, for instance in level of expertise, can require quite distinct interventions (Kalyuga, Ayres, Chandler, \& Sweiler, 2003). Meaningful contextual differences on the other hand, for instance across domains (such as reading and math, or addition and multiplication), may likewise require distinct interventions. Solely assessing the main effect of an intervention, which is usually done in simple A/B tests, will not suffice in an educational context. Rather, it must be assessed how each of the different conditions serve the different individuals and contexts (Savi et al., 2015; Williams et al., 2014).

Once these individual and contextual differences are identified, A/B tests can help determine which learner benefits from which intervention at which moment. This way, either the effect of a global intervention (i.e., targeting all learners in the environment) can be determined with respect to the different individual and contextual characteristics, or the effect of a local intervention (i.e., targeting a relevant subgroup, such as learners with a specific misconception or level of expertise). The former can be considered a grapeshot method, revealing which individual differences are meaningful with respect to the intervention, yet potentially hurting certain subgroups that do not benefit from the intervention or are even harmed by it. On the other hand, the latter is more specific and especially beneficial as it provides the first step 
towards fully adaptive interventions.

A final consideration in tailoring $\mathrm{A} / \mathrm{B}$ tests to online learning is the intervention to choose. As mentioned previously, whereas in traditional website optimization a simple intervention such as coloring the buy button might suffice; optimizing the learning rate of individual learners requires more effort. Fortunately, the learning sciences provide a wealth of knowledge on pedagogical interventions that likely improve achievement and that can be scaled, tested and implemented online. Examples of interventions that have already proven to scale, as diverse as increasing the motivation to learn, optimizing the exerted effort in learning, and providing proper guidance in the learning process, include mindset interventions (Paunesku, Walton, Romero, Smith, Yeager, \& Dweck, 2015), spaced practice (Xiong \& Beck, 2014), and personalized feedback (Piech, Huang, Nguyen, Phulsuksombati, Sahami, \& Guibas, 2015).

\section{Limitations of $\mathbf{A} / \mathbf{B}$ Tests}

Measuring up to the requirements discussed above will enable the full potential of $\mathrm{A} / \mathrm{B}$ tests in online learning, however its opportunities naturally have limitations. The first is its online nature. For instance, most online learning settings allow control over delivery of content and interactions with technology, but do not allow for tight control over the amount and time of study; students learn at their own pace and time. Allied to that, students may receive additional education that enhances similar abilities that are trained in the online environment. Also, online learning likely has a selection effect; students using learning online might not be representative of the total population of students (e.g., due to self-selection). These problems arise due to the in vivo nature of $\mathrm{A} / \mathrm{B}$ tests. Although randomization and large subject pools may eliminate possible bias coming from the treatment assignment, heterogeneity likely is large and in order to secure high internal validity one must anticipate serious challenges (similar and other challenges 
of A/B tests are for instance discussed by Kohavi et al., 2009, Kohavi et al., 2014, and Lu and Liu, 2014).

Also, as mentioned previously, whereas comparisons in traditional (offline) RCTs can go across institutions and cover distinctly different educational methods that may or may not include online components, $\mathrm{A} / \mathrm{B}$ tests are limited to a single online platform. Typical A/B tests are therefore limited in breadth and support mainly incremental steps, ultimately facing the risk of ending up in a local maximum (i.e., optimal learning given the constraints of the platform that is used). Although offline RCTs and A/B tests thus utilize the same principals, they do serve quite distinct goals. A/B tests are most appropriate to identify the interventions that contribute to the development of an effective platform for learning, are less suited to the comparison of entirely different online educational frameworks, and naturally fail if comparisons are made with traditional (offline) learning. Thus, although performing offline RCTs in online learning remains a serious challenge (Lack, 2013), they do complement A/B tests and fill a gap.

Another gap that $\mathrm{A} / \mathrm{B}$ tests fail to fill is the vast amount of non-experimental data online learning accumulates, which is complementary to experiments. Learning data, from demographics to activity measures and error patterns, provide a rich source of (nonexperimental) information and may also guide the development of effective platforms for learning (Long \& Siemens, 2011). For example, it may reveal the level of expertise or typical misconceptions of a learner. As discussed previously, accurate identification of such expertise or misconceptions may in turn enable the use of increasingly personalized interventions. Machine learning provides powerful data mining techniques that are most commonly used for this purpose. The combination of these exploratory techniques and confirmatory A/B tests may proof particularly powerful, since hypotheses can be generated from exploring the non-experimental 
data and A/B tests can experimentally decide which hypotheses are indeed fertile.

Whereas the previous limitations result from technical limitations of online experimentation, we end with an ethical consideration. Although online experimentation is common (Christian, 2012), some A/B tests have been received with mixed reviews. For instance, in 2014 complaints were filed against Facebook for a large-scale experiment they performed on emotional contagion, and European privacy regulators are examining the case (Goel, 2014). Within the domain of learning there too is a longstanding debate among proponents and critics of experimentation. Naturally, Institutional Review Boards watch over the boundaries of what is generally accepted. But although traditional educational institutions too are starting to acknowledge the desirable effects of experimentation (e.g., Coughlan, 2014), the controversy around the Facebook study does demonstrate a serious societal call to consider clear ethical guidelines. Since the emergence of online experimentation is recent, these guidelines need to be actively developed and shaped, and one may anticipate changes.

\section{Discussion}

Although online learning has already made its promise of being highly accessible and affordable, its unsatisfactory effectiveness seriously constrains its full potential. The discussed experimental approach, notwithstanding its limitations, provides a powerful opportunity to increase its effectiveness and thus promises to provide a much-desired leap forward. Furthermore, and most importantly, the insights and priorities that arose from this overview and synthesis of A/B tests in large-scale online learning should help advance and direct future research in this field, enabling its many benefits.

First of all, consulting the different learning sciences and translating robust interventions to online environments, may already greatly improve online education. Second, whereas too 
commonly so-called 'B tests' are used (i.e., blindly adopting an intervention without verifying its effectiveness in a randomized experiment, and thereby taking the risk of unknowingly introducing detrimental interventions), A/B tests help increase an intervention's impact by not only verifying its effectiveness, but also enabling subsequent tweaking in order to optimize the effect, and thus by making sure it is evidence-based. As a step beyond typical laboratory experiments external validity is maximized, since the interventions are evaluated on exactly the platform that is used for the actual learning (Brinkhuis et al., 2015). Finally, tailoring these interventions to each individual learner will most likely be one of the biggest challenges, but likewise has great potential for increasing the return on investment of online learning.

An experimental approach also provides an opportunity to answer a widely-heard call for more research on the effective components of online learning (e.g., Means, Bakia, \& Murphy, 2014). Despite the rapid growth and adoption of online learning, less evidence has surfaced on what these components are. As it appears that more A/B tests are actually conducted than being reported, we encourage both academic and corporate researchers in online learning to share their data, and disseminate and replicate their experimental (null) findings (Makel \& Plucker, 2014, showed that replications are scarce in educational research) (Franco, Malhotra, \& Simonovits, 2014, showed that null results are often not written up and submitted). With the use of for instance the 'conceptual framework for describing online learning' (Means et al., 2014), these findings will all help build an evidence-based body of knowledge on effective components in online learning.

Moreover, as an additional benefit A/B tests are an excellent tool for triangulation. Not only can findings corroborate for instance laboratory experiments and classroom observations, their large-scale, double-blinding, and ecological validity offer a distinct means to discern causal 
effects in the learning sciences. Indeed, the problems encountered in offline educational RCTs are profound: a double-blind procedure and strict randomization, essential ingredients of proper randomized controlled experimentation, are often impracticable. This is particularly problematic since large sample sizes are notoriously difficult to obtain while effect sizes of learning interventions are often small. The online environment helps address these issues, as large sample sizes are relatively easy to obtain, enabling a greater sensitivity to relatively small effect sizes and generally more reliable results.

One-to-one tutoring and its attributed effectiveness might remain an eternal ambition that is never fully achieved, but it does sets the ultimate challenge and surely can be approached by accurately and continuously assessing learners, and tailoring the learning interventions to each and every individual. In the current paper, we have focused on one complimentary approach, that is broadly applicable and minimally invasive yet is only to a limited extent adopted by the online learning community, and moreover receives little attention in the larger learning community. A/B testing is an online-only and within-platform approach that enables evidencebased iterative improvement of online learning. Deploying A/B tests in online learning however requires us to move away from aiming interventions at the general population of learners, and to seriously take into account the intricate nature of not only learning in general, but also the inseparable ecological online environment, and the individual and contextual differences. A/B tests, when deployed correctly, provide a powerful opportunity that may help determine the effective components in online learning and eventually contribute to an increase in return on investment.

\section{References}

Allen, I. E., \& Seaman, J. (2014, January). Grade change: Tracking online education in the 
United States. Retrieved from http://onlinelearningconsortium.org

Aud, S., Hussar, W., Johnson, F., Kena, G., Roth, E., Manning, E., ... Notter, L. (2012, May 24). The condition of education 2012. Retrieved from National Center for Education Statistics website: http://nces.ed.gov

Barnett, S. M., \& Ceci, S. J. (2002). When and where do we apply what we learn? A taxonomy for far transfer. Psychological Bulletin, 128, 612-637. doi:10.1037/0033-2909.128.4.612

Bloom, B. S. (1984). The 2 sigma problem: The search for methods of group instruction as effective as one-to-one tutoring. Educational Researcher, 13, 4-16. doi:10.3102/0013189X013006004

Brinkhuis, M. J. S., Savi, A. O., Coomans, F., Hofman, A. D., van der Maas, H. L. J., \& Maris, G. (2015). Learning as it happens: Advances in computerized adaptive practice. Manuscript submitted for publication.

Chafkin, M. (2013, November 14). Udacity's Sebastian Thrun, godfather of free online education, changes course. Fast Company. Retrieved from http://www.fastcompany.com Christian, B. (2012, April 25). The A/B test: Inside the technology that's changing the rules of business. Wired. Retrieved from http://www.wired.co.uk

Content Experiments. (n.d.). Retrieved from http://edx-open-learningxml.readthedocs.org/en/latest/content-experiments/index.html

Coughlan, S. (2014, June 11). School appoints 'head of research'. BBC News. Retrieved from http://www.bbc.com/news

Coursera. (2014a, May 17). Government of Trinidad and Tobago works with Coursera to boost education and improve career skills nationwide. Retrieved from http://blog.coursera.org Coursera. (2014b, June 17). Singapore government builds training program around Johns 
Hopkins University data science specialization. Retrieved from http://blog.coursera.org

Fox, P. (2014, March 3). A/B testing curriculum: To sneak peek or not? [Web log post].

Retrieved from http://cs-blog.khanacademy.org/

Franco, A., Malhotra, N., \& Simonovits, G. (2014). Publication bias in the social sciences:

Unlocking the file drawer. Science, 345, 1502-1505. doi:10.1126/science.1255484

Ginder, S., \& Stearns, C. (2014, June 2). Enrollment in distance education courses, by state: Fall 2012. Retrieved from National Center for Education Statistics website: http://nces.ed.gov

Goel, V. (2014, July 2). After uproar, European regulators question Facebook on psychological testing. The New York Times. Retrieved from http://www.nytimes.com

Greenberg, B., Medlock, L., \& Stephens, D. (2011, December 6). Lessons learned from a blended learning pilot. Retrieved from http://www.blendmylearning.com

Greenhow, C., Robelia, B., \& Hughes, J. E. (2009). Learning, teaching, and scholarship in a digital age: Web 2.0 and classroom research: What path should we take now?

Educational Researcher, 38, 246-259. doi:10.3102/0013189X09336671

Heffernan, N. T., \& Heffernan, C. L. (2014). The ASSISTments ecosystem: Building a platform that brings scientists and teachers together for minimally invasive research on human learning and teaching. International Journal of Artificial Intelligence in Education, 24, 470-497. doi:10.1007/s40593-014-0024-X

Hu, D. (2011). How Khan Academy is using machine learning to assess student mastery. Retrieved from http://david-hu.com/2011/11/02/how-khan-academy-is-using-machinelearning-to-assess-student-mastery.html

ICDE. (2013, December 18). An international outlook on distance education: Global online higher education report, GlobalOHER. Retrieved from International Council for Open 
and Distance Education website: http://www.icde.org

Jaggars, S. S., Edgecombe, N., \& Stacey, G. W. (2013, April). What we know about online course outcomes. Retrieved from Columbia University, Community College Research Center website: http://ccrc.tc.columbia.edu

Johnson, H., \& Mejia, M. C. (2014, May). Online learning and student outcomes in community colleges. Retrieved from Public Policy Institute of California website: http://www.ppic.org

Kalyuga, S., Ayres, P., Chandler, P., \& Sweiler, J. (2003). The expertise reversal effect. Educational Psychologist, 38, 23-31. doi:10.1207/S15326985EP3801_4

Kena, G., Aud, S., Johnson, F., Wang, X., Zhang, J., Rathbun, A., . . Rosario, V. (2014, May 28). The condition of education 2014. Retrieved from National Center for Education Statistics website: http://nces.ed.gov

Klinkenberg, S., Straatemeier, M., \& van der Maas, H. L. J. (2011). Computer adaptive practice of maths ability using a new item response model for on the fly ability and difficulty estimation. Computers \& Education, 57, 1813-1824. doi:10.1016/j.compedu.2011.02.003

Kohavi, R., Deng, A., Longbotham, R., \& Xu, Y. (2014). Seven rules of thumb for web site experimenters. Retrieved from http://www.expplatform.com/Pages/SevenRulesofThumbforWebSiteExperimenters.aspx

Kohavi, R., Longbotham, R., Sommerfield, D., \& Henne, R. M. (2009). Controlled experiments on the web: Survey and practical guide. Data Mining and Knowledge Discovery, 18, 140-181. doi:10.1007/s10618-008-0114-1

Kolowich, S. (2014, January 16). Exactly how many students take online courses? The Chronicle of Higher Education. Retrieved from http://chronicle.com 
Lack, K. A. (2013, March 21). Current status of research on online learning in postsecondary education. Retrieved from http://sr.ithaka.org

Lewin, T. (2012, July 17). Consortium of colleges takes online education to new level. The New York Times. Retrieved from http://www.nytimes.com

Long, P., \& Siemens, G. (2011, September/October). Penetrating the fog: Analytics in learning and education. Educause Review, 46, 31-40.

Lu, L., \& Liu, C. (2014). Separation strategies for three pitfalls in a/b testing. Retrieved from http://www.ueo-workshop.com/wp-content/uploads/2014/04/Separation-strategies-forthree-pitfalls-in-AB-testing_withacknowledgments.pdf

Makel, M. C. \& Plucker, J. A. (2014). Facts are more important than novelty: Replication in the education sciences. Educational Researcher, 43, 304-316.

doi:10.3102/0013189X14545513

Means, B., Bakia, M., \& Murphy, R. (2014). Learning online: What research tells us about whether, when and how. New York: Routledge.

Molnar, M. (2013, August 30). iPad-centered 'Steve Jobs schools' open in The Netherlands. Education Week. Retrieved from http://www.edweek.org

Murphy, R., Gallagher, L., Krumm, A., Mislevy, J., \& Hafter, A. (2014). Research on the use of Khan Academy in schools: Research brief. Retrieved from http://www.sri.com/work/publications

NCES. (2012, July 10). Ipeds changes for 2012-13 relating to distance education. Retrieved from National Center for Education Statistics website: http://nces.ed.gov/ipeds

Ng, A. (2014, May 16). A personal message from co-founder Andrew Ng. Retrieved from http://blog.coursera.org 
Norman, G. (2003). RCT = results confounded and trivial: The perils of grand educational experiments. Medical Education, 37, 582-584. doi:10.1046/j.1365-2923.2003.01586.x

Novet, J. (2013, November 19). Coursera improves online education using - you guessed itdata. Retrieved from http://venturebeat.com

Oncu, S., \& Cakir, H. (2011). Research in online learning environments: Priorities and methodologies. Computers \& Education, 57, 1098-1108. doi:10.1016/j.compedu.2010.12.009

Paunesku, D., Walton, G. M., Romero, C., Smith, E. N., Yeager, D. S., \& Dweck, C. S. (2015). Mind-set interventions are a scalable treatment for academic underachievement. Psychological Science, 26, 784-793. doi:10.1177/0956797615571017

Piech, C., Huang, J., Nguyen, A., Phulsuksombati, M., Sahami, M., \& Guibas, L. (2015). Learning program embeddings to propagate feedback on student code. arXiv preprint arXiv:1505.05969

Reich, J. (2015). Rebooting MOOC research. Science, 347, 34-35. doi:10.1126/science.1261627

Rowan, D. (2013, August 27). Online education is redefining learning itself, says Khan Academy founder. Wired. Retrieved from http://www.wired.co.uk

Savi, A. O., van der Maas, H. L. J., \& Maris, G. K. J. (2015). Navigating massive open online courses. Science, 347, 958. doi:10.1126/science.347.6225.958

Selwyn, N. (2000). Researching computers and education - glimpses of the wider picture. Computers \& Education, 34, 93-101. doi:10.1016/S0360-1315(00)00006-3

Siegler, R. S. (1994). Cognitive variability: A key to understanding cognitive development. Current Directions in Psychological Science, 3, 1-5. doi:10.1111/1467-8721.ep10769817

Siegler, R. S., \& Crowley, K. (1991). The microgenetic method: A direct means for studying 
cognitive development. American Psychologist, 46, 606-620. doi:10.1037//0003066X.46.6.606

Simonite, T. (2013, June 5). As data floods in, massive open online courses evolve. Retrieved from http://www.technologyreview.com

Sims, Z. (2014, April 23). We're learning too: A new codecademy. Retrieved from http://www.codecademy.com/blog

Slavin, R. E. (2002). Evidence-based education policies: Transforming educational practice and research. Educational Researcher, 31, 15-21. doi:10.3102/0013189X031007015

Stevenson, S. (2014, January 26). How do you say addictive in Spanish? Retrieved from http://www.slate.com

Takeuchi, L. M., \& Vaala, S. (2014). Level up learning: A national survey on teaching with digital games. Retrieved from http://www.joanganzcooneycenter.org

Ungerleider, N. (2014, April 23). How Duolingo uses A/B testing to understand the way you learn. Fast Company. Retrieved from http://www.fastcompany.com

VanLehn, K. (2011). The relative effectiveness of human tutoring, intelligent tutoring systems, and other tutoring systems. Educational Psychologist, 46, 197-221. doi:10.1080/00461520.2011.611369

Vernau, K. \& Hauptmann, M. (2014, April). Corporate learning goes digital: How companies can benefit from online education. Retrieved from http://www.rolandberger.com

Watson, J., Murin, A., Vashaw, L., Butch, G., \& Rapp, C. (2013). Keeping pace with k-12 online and blended learning. Retrieved from http://www.kpk12.com

Williams, J. J., Li, N., Kim, J., Whitehill, J., Maldonado, S., Pechenizkiy, M., .. Heffernan, N. (2014). The MOOClet framework: Improving online education through experimentation 
and personalization of modules. Retrieved from http://ssrn.com/abstract=2523265

Williams, J. J., Paunesku, D., Haley, B., \& Sohl-Dickstein, J. (2013). Measurably increasing motivation in MOOCs. Proceedings of the 1st Workshop on Massive Open Online Courses at the 16th Annual Conference on Artificial Intelligence in Education, Memphis, TN. Abstract retrieved from http://ceur-ws.org/Vol-1009/

Windschitl, M. (1998). The WWW and classroom research: What path should we take? Educational Researcher, 27, 28-33. doi:10.3102/0013189X027001028

Xiong, X., \& Beck, J. (2014). A study of exploring different schedules of spacing and retrieval interval on mathematics skills in ITS environment. In S. Trausan-Matu, K. E. Boyer, M. Crosby, \& K. Panourgia (Eds.), Intelligent tutoring systems (pp. 504-509). Springer International Publishing. 